\title{
The status of assisted reproductive technology in Korea in 2012
}

\author{
Committee for Assisted Reproductive Technology Statistics, Korean Society for Assisted Reproduction, Gyoung Hoon Lee', \\ Hyun Jin Song ${ }^{1}$, Young Min $\mathrm{Choi}^{2,3}$, Hyuck Dong Han ${ }^{4}$
}

${ }^{1}$ I-one Center, Seoul Women's Hospital, Bucheon; ${ }^{2}$ Department of Obstetrics and Gynecology, ${ }^{3}$ The Institute of Reproductive Medicine and Population, Medical Research Center, Seoul National University College of Medicine, Seoul; ${ }^{4}$ Department of Obstetrics and Gynecology, Wonju Severance Christian Hospital, Yonsei University Wonju College of Medicine, Wonju, Korea

\begin{abstract}
Objective: This study was designed to report the status of assisted reproductive technology (ART) therapy in South Korea between January 1 , 2012 and December 31, 2012.

Methods: A localized online survey, originally developed by the International Committee Monitoring Assisted Reproductive Technologies, was first launched and provided to all available ART centers via email in 2015. Fresh embryo transfer (FET) cases were categorized as standard in vitro fertilization, intracytoplasmic sperm injection (ICSI), or half-ICSI. Thawed embryo transfer (TET) and other related procedures, including surgical sperm retrieval, were surveyed.

Results: Data from 33,956 ovum pick-up procedures were provided by 75 clinics in 2012. Of the 33,088 cycles in which ovums were retrieved, a complete transfer was performed in $90.5 \%$ (29,932 cycles). In addition, 10,079 FET cycles were confirmed to have resulted in clinical pregnancy, representing a pregnancy rate of $30.5 \%$ per ovum pick-up and 33.7\% per ET. The most common number of embryos transferred in FET was 2 (41.6\%), followed by $3(34.0 \%)$, and non-elective single ETs (10.0\%). Of the 10,404 TET cycles in which transfer was completed, 3,760 clinical pregnancies (36.1\%) were confirmed by ultrasonography.

Conclusion: The overall clinical pregnancy rate for FET and TET cycles in 2012 was higher than in 2011 (33.7\% vs. 33.2\% and 36.1\% vs. 31.1\%, respectively). The most common number of embryos transferred in FET cycles was 2, unlike in 2011.
\end{abstract}

Keywords: Assisted reproductive techniques; Surveys

\section{Introduction}

Subfertility is being overcome through advancements in fertility treatment, including controlled ovarian stimulation, intrauterine insemination, and in vitro fertilization (IVF). Since the first IVF baby was born in South Korea (hereafter Korea) in 1985, the use of assisted reproductive technology (ART) to overcome infertility has dramatically

Received: Oct 5, 2016 · Revised: Dec 13, 2016 · Accepted: Dec 13, 2016 Corresponding author: Young Min Choi

Department of Obstetrics and Gynecology, The Institute of Reproductive Medicine and Population, Medical Research Center, Seoul National University College of Medicine, 101 Daehak-ro, Jongno-gu, Seoul 03080, Korea Tel: +82-2-2072-2385 Fax:+82-2-762-3599 E-mail:ymchoi@snu.ac.kr

This is an Open Access article distributed under the terms of the Creative Commons Attribution Non-Commercial License (http://creativecommons.org/licenses/by-nc/4.0/) which permits unrestricted non-commercial use, distribution, and reproduction in any medium, provided the original work is properly cited. increased, along with the number of fertility centers and live births of ART-conceived infants. The most recent national statistics indicate that $2.83 \%$ of all births in 2011 were associated with financial support from the National Supporting Program for the Subfertile administered by the Korean Ministry of Health and Welfare (KMHW).

The Korean Society of Obstetrics and Gynecology collected ART data and published their first report in 1992, and the Korean Society of Assisted Reproduction (KSAR) took over that role in 2013. Although the data collection forms are based on the International Committee Monitoring Assisted Reproductive Technologies Tool Box for Data Collection forms, they have been revised to collect a few additional pieces of information starting in 2014; however, the forms were kept similar in order to allow the comparison of results from previous years.

The primary objective of this database was to facilitate a collabora- 
tion between the Committee for ART Statistics and fertility centers in Korea and to obtain an overall national picture of fertility treatments based on data from all ART clinics in Korea that voluntarily provided data on procedures performed between January 2012 and December 2012.

\section{Methods}

\section{Subjects}

Of the 188 ART clinics that were invited to input their data into the module created by the Committee of Statistics of the KSAR, 75 reported their outcomes (return rate, 39.8\%). The complete list of the ART clinics that participated in this survey is listed by region in Supplement 1.

\section{Methods}

A revised Korean-language online survey was provided by email to all ART clinics registered with the KMHW. Responses were collected online (http://www.ivfkorea.or.kr). The survey was originally based on the International Committee Monitoring Assisted Reproductive Technologies Tool Box for Data Collection forms (Supplement 2).

The IVF data were also categorized according to outcomes after embryo transfer (ET) in fresh cycles, after thawed ET, and in association with other related procedures, as in the previous survey. Thereafter, the data were subclassified according to the IVF method, such as conventional IVF, intracytoplasmic sperm injection (ICSI), and half-ICSI.

All data reflected treatment cycles that took place in 2012. However, the precise characteristics of the starting and ending day of the data submitted (ovum pick-up day or controlled ovarian stimulation day) were left to the clinic's discretion, thereby resulting in a mixed dataset. Clinical pregnancy was defined as the presentation of an intrauterine gestational sac on ultrasound examination. Cases of biochemical pregnancy were excluded if elevated serum beta-human chorionic gonadotropin was the only sign of pregnancy. Live birth delivery was defined as the birth of one or more live infants, with the delivery of multiple infants counting as a single live-birth delivery.

\section{Analysis}

The contribution of ART to each outcome was calculated by dividing the total number of outcomes by the number of ovum pick-ups and the number of ETs. All data were analyzed using PASW ver. 18.0 (SPSS Inc., Chicago, IL, USA).

\section{Results}

\section{Overview}

\section{1) Data provided by the fertility clinics}

Seventy-five centers reported 33,956 ovum pick-up cycles that were initiated in 2012. In total, 41,995 ET cycles were reported.

\section{2) Types of ART procedures}

Fresh ET was used in approximately $71.3 \%(n=29,932)$ of the 41,995 ART cycles that were reported. ART cycles that used frozen embryos were the next most common type of procedure, accounting for approximately $24.9 \%$ of the total ART cycles $(n=10,449)$. The less common procedures were natural-cycle IVF $(n=747)$, in vitro maturation $(n=414)$, preimplantation genetic diagnosis $(n=311)$, and the surgical retrieval of sperm (Table 1).

\section{3) Trends by age}

Women 30 to 34 years of age comprised the majority (38.7\%) of all fresh ET cycles performed in 2012. The age distribution among patients who received fresh ET cycles was as follows: $36.0 \%$ among

Table 1. The number of cycles for each ART procedure

\begin{tabular}{lcc}
\hline Type of ART procedure & No. of cycles in 2012 & No. of cycles in 2011 \\
\hline Fresh ET & $29,932(71.3)$ & $27,683(74.8)$ \\
Thawed ET & $10,449(24.9)$ & $8,826(23.9)$ \\
Others ${ }^{\text {a) }}$ & $1,614(3.8)$ & $481(1.3)$ \\
Total & $41,995(100.0)$ & $36,990(100.0)$ \\
\hline
\end{tabular}

Values are presented as number (\%).

ART, assisted reproductive technology; ET, embryo transfer.

a) The category of "others" includes preimplantation genetic diagnosis/screening, natural in vitro fertilization, ovum donation, and in vitro maturation cycles.

Table 2. The number of fresh ET and thawed ET cycles by age group

\begin{tabular}{|c|c|c|c|c|}
\hline \multirow{2}{*}{ Age group (yr) } & \multicolumn{2}{|c|}{ Fresh ET } & \multicolumn{2}{|c|}{ Thawed ET } \\
\hline & 2012 & 2011 & 2012 & 2011 \\
\hline$\leq 29$ & $1,848(6.2)$ & $1,946(7.1)$ & $803(7.7)$ & $717(8.4)$ \\
\hline $30-34$ & $11,573(38.7)$ & $10,416(38.0)$ & $4,817(46.3)$ & $3,802(44.5)$ \\
\hline $35-39$ & $10,782(36.0)$ & 10,029 (36.6) & $3,718(35.7)$ & $3,039(35.6)$ \\
\hline$\geq 40$ & $5,713(19.1)$ & $5,002(18.3)$ & $1,066(10.2)$ & $978(11.5)$ \\
\hline Total & $29,916^{\text {a) }}(100.0)$ & $27,396(100.0)$ & $10,404^{\mathrm{a})}(100.0)$ & $8,536(100.0)$ \\
\hline
\end{tabular}

Values are presented as number (\%).

$\mathrm{ET}$, embryo transfer.

a)The patient's age was missing for 16 fresh ET cycles and 45 thawed ET cycles. 
those 35 to 39 years of age, $19.1 \%$ among those over 40 years of age, and $6.2 \%$ among those 25 to 29 years of age. Similarly, women aged 30 to 34 years of age comprised the largest group of women who underwent thawed ET cycles, accounting for $46.3 \%$ of those cycles. Women 35 to 39 years of age comprised the next most common age group (Table 2).

\section{4) Clinical pregnancy according to age}

Among the patients from whom ovums were retrieved $(n=$ $33,088), 90.5 \%$ ( $n=29,932)$ successfully transferred their embryos in 2012. Of the 29,916 cycles for which the patient's age was reported, 10,079 patients were confirmed to have achieved clinical pregnancy. Among the patients who underwent thawed ET $(n=10,404), 3,760$ patients were confirmed to have achieved clinical pregnancy, representing a $36.1 \%$ clinical pregnancy rate per ET cycle. The clinical pregnancy rate per ET of fresh cycles was higher among patients 34 years of age or younger than the corresponding clinical pregnancy rate per ET for thawed ET cycles (Table 3).

\section{5) Live deliveries by treatment type}

Although many of the clinics that participated in this study may not have been able to follow their pregnant patients through the actual delivery, live births were reported for a total of 5,467 patients. However, several clinics did not report the number of live births or whether they were accomplished by IVF, ICSI, half-ICSI, or thawed ET in 2012 (Table 4).

\section{Fresh cycles \\ 1) Clinical pregnancy by fertilization method}

Of the 29,932 fresh ET cycles, $34.5 \%$ used embryos obtained by IVF, $45.6 \%$ by ICSI, and $20.0 \%$ by half-ICSI.

Among the cycles in which embryos were successfully transferred, the clinical pregnancy rate was $36.9 \%, 28.7 \%$, and $39.7 \%$ among those who underwent IVF, ICSI, and half-ICSI procedures, respectively (Table 5).

Table 3. Clinical outcomes by age group

\begin{tabular}{|c|c|c|c|c|c|c|c|c|c|c|}
\hline \multirow{2}{*}{ Outcome } & \multicolumn{5}{|c|}{ Fresh cycle (yr) } & \multicolumn{5}{|c|}{ Thawed cycle (yr) } \\
\hline & $\leq 29$ & $30-34$ & $35-39$ & $\geq 40$ & All & $\leq 29$ & $30-34$ & $35-39$ & $\geq 40$ & All \\
\hline ET cycles (n) & 1,848 & 11,573 & 10,782 & 5,713 & 29,916 & 803 & 4,817 & 3,718 & 1,066 & 10,404 \\
\hline Clinical pregnancy (n) & 834 & 4,782 & 3,543 & 920 & 10,079 & 356 & 1,884 & 1,273 & 247 & 3,760 \\
\hline Delivery reported (n) & 337 & 1,973 & 1,386 & 295 & 3,991 & 159 & 773 & 518 & 73 & 1,523 \\
\hline CPR per ET (\%) & 45.1 & 41.3 & 32.9 & 16.1 & 33.7 & 44.3 & 39.1 & 34.2 & 23.2 & 36.1 \\
\hline
\end{tabular}

ET, embryo transfer; CPR, clinical pregnancy rate.

Table 4. Live births by fertilization method

\begin{tabular}{lrcccr}
\hline $\begin{array}{l}\text { Fertilization } \\
\text { method }\end{array}$ & Singleton & $\begin{array}{c}\text { Twin } \\
\text { delivery }\end{array}$ & $\begin{array}{c}\text { Triplet } \\
\text { delivery }\end{array}$ & $\begin{array}{c}\text { Quadruplet or } \\
\text { more delivery }\end{array}$ & Total \\
\hline IVF & 1,007 & 331 & 1 & 0 & 1,339 \\
ICSI & 1,274 & 409 & 9 & 0 & 1,692 \\
Half-ICSI & 642 & 265 & 4 & 0 & 911 \\
Thawed ET & 1,116 & 398 & 11 & 0 & 1,525 \\
Total & 4,309 & 1,403 & 25 & 0 & 5,467 \\
\hline
\end{tabular}

Values are presented as number.

IVF, in vitro fertilization; ICSI, intracytoplasmic sperm injection; ET, embryo transfer.
Table 5. Clinical pregnancies according to fertilization method in fresh cycles

\begin{tabular}{lcccc}
\hline Outcome & IVF & ICSI & Half-ICSI & All \\
\hline ET, $n(\%)$ & $10,319(34.5)$ & $13,635(45.6)$ & $5,978(20.0)$ & $29,932(100.0)$ \\
$\begin{array}{l}\text { Clinical } \\
\text { pregnancy (n) }\end{array}$ & 3,804 & 3,918 & 2,375 & 10,097 \\
CPR per ET (\%) & 36.9 & 28.7 & 39.7 & 33.7 \\
\hline
\end{tabular}

IVF, in vitro fertilization; ICSI, intracytoplasmic sperm injection; ET, embryo transfer; CPR, clinical pregnancy rate.

Table 6. Clinical pregnancies by the number of transferred embryos in fresh cycles

\begin{tabular}{|c|c|c|c|c|c|c|c|c|}
\hline \multirow{3}{*}{ Outcome } & \multicolumn{8}{|c|}{ No. of embryos transferred } \\
\hline & \multicolumn{2}{|c|}{1} & \multirow{2}{*}{2} & \multirow{2}{*}{3} & \multirow{2}{*}{4} & \multirow{2}{*}{5} & \multirow{2}{*}{6 or more } & \multirow{2}{*}{ Total } \\
\hline & Non-eSET & eSET & & & & & & \\
\hline ET cycles $(n, \%)$ & $3,004(10.0)$ & $1,499(5.0)$ & $12,457(41.6)$ & $10,180(34.0)$ & $2,469(8.2)$ & $303(1.0)$ & $20(0.1)$ & $29,932(100.0)$ \\
\hline Clinical pregnancy (n) & 596 & 324 & 4,715 & 3,409 & 775 & 54 & 4 & 9,877 \\
\hline CPR per ET (\%) & 19.8 & 21.6 & 37.9 & 33.5 & 31.4 & 17.8 & 20 & 32.9 \\
\hline Two or more gestational sacs ( $\mathrm{n}$ ) & 6 & 5 & 1,288 & 992 & 256 & 15 & 2 & 2,564 \\
\hline MPR per ET (\%) & 0.2 & 0.3 & 10.3 & 9.7 & 10.2 & 5 & 10 & 8.6 \\
\hline
\end{tabular}

eSET, elective single embryo transfer; ET, embryo transfer; CPR, clinical pregnancy rate; MPR, multiple pregnancy rate. 


\section{2) Clinical pregnancy by the number of transferred embryos}

Of the 29,932 fresh embryo cycles, $75.6 \%$ were 2-ET or 3-ET cycles (2-ET, $41.6 \% ; 3-\mathrm{ET}, 34.0 \%$ ), and $15.0 \%$ were single ETs (elective single $\mathrm{ET}, 5.0 \%$; non-elective single ET, 10.0\%). Overall, the clinical pregnancy rate per ET was $37.9 \%$ for 2-ET cycles and 33.5\% for 3-ET cycles.

The multiple pregnancy rate per ET was the highest among women who underwent 2-ET cycles (10.3\%), followed by women who underwent 4-ET cycles (10.2\%) (Table 6).

\section{Thawing cycles}

\section{1) Clinical pregnancy by fertilization method}

Of the 10,408 thawed ET cycles, $44.5 \%$ used IVF and $55.5 \%$ used ICSI or half-ICSI. The clinical pregnancy rate was $35.8 \%$ for the IVF group, and $36.4 \%$ for the half-ICSI group (Table 7).

\section{2) Clinical pregnancy by the number of transferred embryos}

Of the 10,430 thawed ET cycles, $76.0 \%$ were 2 -ET or 3-ET cycles (2-

Table 7. Clinical pregnancies by the fertilization method in frozenthawed ET cycles

\begin{tabular}{lccc}
\hline \multirow{2}{*}{ Outcome } & \multicolumn{3}{c}{ Thawed ET } \\
\cline { 2 - 4 } & IVF & ICSI & Total \\
\hline ET cycles (n, \%) & $4,633(44.5)$ & $5,775(55.5)$ & $10,408(100.0)$ \\
Clinical pregnancy (n) & 1,657 & 2,103 & 3,760 \\
CPR per ET (\%)) & 35.8 & 36.4 & 36.1 \\
\hline
\end{tabular}

ET, embryo transfer; IVF, in vitro fertilization; ICSI, intracytoplasmic sperm injection; CPR, clinical pregnancy rate.

a)The total number of thawed ET cycles was 10,449. Data on the fertilization method were missing for 41 patients.
ET, 49.2\%; 3-ET, 26.8\%), and 16.5\% were single ETs. Overall, the clinical pregnancy rate per ET was $38.4 \%$ for 2-ET cycles and $38.6 \%$ for 3-ET cycles.

The multiple pregnancy rate per ET was the highest among women who underwent $6+$-ET cycles (14.3\%), followed by women who underwent 3-ET cycles (13.6\%) (Table 8).

\section{Other related procedures \\ 1) Surgical retrieval of sperm}

A total of 1,172 cases of surgical sperm retrieval were reported to have successfully progressed to the ET stage. The overall clinical pregnancy rate per ET was 31.5\% (Table 9).

\section{2) Preimplantation genetic diagnosis and preimplantation genet- ic screening}

Of the 311 cycles of preimplantation genetic diagnosis that were planned, a total of 42 cases successfully progressed to clinical pregnancy after ET (283 cycles). In 2012, a total of 2,438 embryos were examined at the preimplantation stage, and 1,157 of these embryos were diagnosed as normal.

Of the 24 cycles of preimplantation genetic screening that were planned, nine cases successfully progressed to clinical pregnancy after ET (21 cycles). In 2012, a total of 185 embryos were examined at the preimplantation stage, and 65 of these embryos were diagnosed as normal.

\section{3) Ovum cryopreservation and ovum donation}

In 2012, ovum cryopreservation was carried out in 111 cycles. Forty cycles were reported to be planned for medical causes and 44 cycles

Table 8. Clinical pregnancies by the number of transferred embryos in frozen-thawed ET cycles

\begin{tabular}{|c|c|c|c|c|c|c|c|}
\hline \multirow{2}{*}{ Outcome } & \multicolumn{7}{|c|}{ No. of frozen-thawed embryos transferred } \\
\hline & One & Two & Three & Four & Five & Six or more & Total \\
\hline ET cycles, $n(\%)^{a)}$ & $1,718(16.5)$ & $5,132(49.2)$ & $2,797(26.8)$ & $718(6.9)$ & $58(0.6)$ & $7(0.1)$ & $10,430(100.0)$ \\
\hline CPR per ET (\%) & 24.4 & 38.4 & 38.6 & 38.9 & 36.2 & 28.6 & 36.2 \\
\hline Two or more gestational sacs ( $\mathrm{n}$ ) & 16 & 547 & 379 & 94 & 4 & 1 & 1,041 \\
\hline MPR per ET (\%) & 0.9 & 10.7 & 13.6 & 13.1 & 6.9 & 14.3 & 10 \\
\hline
\end{tabular}

$\mathrm{ET}$, embryo transfer; CPR, clinical pregnancy rate; MPR, multiple pregnancy rate.

a)The total number of thawed ET cycles was 10,449. Data on the number of frozen thawed ET were missing for 19 patients.

Table 9. Outcomes of the surgical retrieval of sperm

\begin{tabular}{lccccc}
\hline Technique & OPU cycle & Transfer cycle & Clinical pregnancy & Live birth & CPR per ET (\%) \\
\hline PESA & 53 & 49 & 14 & 2 & 28.6 \\
TESE & 1,119 & 782 & 248 & 119 & 31.7 \\
Total & 1,172 & 831 & 262 & 121 & 31.5 \\
\hline
\end{tabular}

Values are presented as number unless otherwise indicated.

OPU, ovum pick-up; CPR, clinical pregnancy rate; ET, embryo transfer; PESA, percutaneous sperm aspiration; TESE, testicular sperm extraction. 
for social causes. In 2012, ovum donation was performed in 480 cycles. Of these, 418 cycles were successfully transferred, resulting in clinical pregnancy in 87 cycles and live births in 35 cycles.

\section{4) In vitro maturation}

A total of 414 cycles using in vitro maturation were reported in 2012. From these cycles, 131 clinical pregnancies and 70 live births were reported.

\section{5) Natural-cycle IVF}

A total of 747 cycles using natural-cycle IVF were reported in 2012. Of these, 43 clinical pregnancies and 18 live births were reported after ET (387 cycles).

\section{6) Selective fetal reductions}

In 2012, 255 selective fetal reductions were reported. The majority of selective fetal reductions were performed in triplet pregnancies, resulting in twin pregnancies (168 cases).

\section{Intrauterine insemination}

Of the 29,144 cycles of intrauterine inseminations, 3,434 cases successfully progressed to clinical pregnancy ( $11.7 \%$ per cycle).

\section{Discussion}

Since the first report was issued in 1995, the number of ART procedures and the pregnancy rate have increased annually in Korea. Although the clinical pregnancy rate per ET in fresh IVF cycles slightly increased from 2011 (33.3\%) to 2012 (33.7\%), these figures should not be applied to a particular couple without considering all of the relevant factors.

The 40,320 ET cycles performed in 2012 resulted in 3,991 live births from 29,916 fresh ET cycles and 1,523 live births from 10,404 thawed ET cycles (including data on embryos that might have been frozen in 2011, but were thawed and transferred in 2012).

This survey suggests that factors relating to the infertile couples (e.g., the woman's age) and procedural factors (e.g., the number of embryos transferred) played an important role in the success of ART treatments. However, the findings in this report are subject to some serious limitations. First, the data submitted were reported by each ART clinic voluntarily and were not validated by any third parties.
Furthermore, the definitions of infertility and subfertility may vary from clinic to clinic [1]. Second, data about patient demographics, medical history, and infertility diagnoses were absent. Third, these data were not linked to the individual patients in whom the procedures were performed. The data do not indicate whether cycles were performed in women receiving ART treatment for the first time or in women who had previously undergone an unsuccessful ART cycle.

In this survey of voluntarily submitted data from 2012, we report the national trends in ART centers in Korea. We hope that this information will reduce the informational gap between infertile couples and ART clinics and serve as a reference for quality control and the self-promotion of ART centers in Korea.

\section{Conflict of interest}

No potential conflict of interest relevant to this article was reported.

\section{Acknowledgments}

We are deeply grateful to those who contributed to the development of this report and helped collect the 2012 ART data. We believe that their assistance in developing this report will be greatly appreciated by future patients who consider undergoing an ART procedure (Supplement 1).

\section{Supplementary materials}

Supplement 1. Seventy-five in vitro fertilization centers participated in the 2012 survey. Supplemental data can be found at: http:// ecerm.org/src/sm/cerm-44-47-s001.pdf.

Supplement 2. The revised Korean-language online survey form used in 2012. Supplemental data can be found at: http://ecerm.org/ src/sm/cerm-44-47-s002.pdf.

\section{Reference}

1. Zegers-Hochschild F, Adamson GD, de Mouzon J, Ishihara O, Mansour R, Nygren $\mathrm{K}$, et al. International Committee for Monitoring Assisted Reproductive Technology (ICMART) and the World Health Organization (WHO) revised glossary of ART terminology, 2009. Fertil Steril 2009;92:1520-4. 